\title{
Nonlinear dynamics in a Cournot duopoly with isoelastic demand
}

\author{
Luciano Fanti • Luca Gori • Mauro Sodini
}

\begin{abstract}
This paper analyses the dynamics of a nonlinear Cournot duopoly with general isoelastic demand (quasi-linear preferences) and quantity-setting firms that have incomplete information about the market demand. Unlike existing papers, we propose a model where the price elasticity of demand is different from one. This causes interesting local and global dynamic events that cannot be observed in the case of unit-elastic demand and homogeneous players. In particular, the global behaviour of the map is studied through the critical curves technique, and numerical simulations show coexistence of attractors, coordination failures and complex structures of the basins of attraction.
\end{abstract}

Keywords Cournot; Isoelastic demand; Nonlinear dynamics

JEL Classification C62; D43; L13

\section{Introduction}

Analysing nonlinear dynamics in oligopoly models dates back at least to Puu [21], later followed, amongst others, by Kopel [18], Puu [22, 23] and Bischi et al. [6, 7, 9]. These papers have renewed interest in the study of expectations formation mechanisms differing from the rational expectations paradigm, as claimed by Agliari et al. [1, p. 527]: "When one takes into account the fact that nonlinear dynamical systems can produce dynamic paths that are not so regular and predictable, one of the major arguments against adaptive expectations does not seem so strong." For instance, Bischi et al. [8] consider a Cournot duopoly with profitmaximising firms and incomplete information that estimate a linear demand function. In particular, they compare the behaviour of firms that use the Local Monopolistic Approximation adjustment process with the behaviour of firms that use an adjustment mechanism based on the Best Reply dynamics (where firms are assumed to have complete knowledge of the market demand with naïve expectations). They show, for the case of unitelastic demand, that less information causes more stability to occur.

L. Fanti

Department of Economics and Management, University of Pisa, Via Cosimo Ridolfi, 10, I-56124 Pisa (PI), Italy

e-mail: lfanti@ec.unipi.it or fanti.luciano@gmail.com

tel.: +390502216369; fax: +390502210603

L. Gori (corresponding author)

Department of Law, University of Genoa, Via Balbi, 30/19, I-16126 Genoa (GE), Italy

e-mail: luca.gorieunige.it or dr.luca.goriegmail.com

tel.: +390102099503; fax: +390102095536

M. Sodini

Department of Economics and Management, University of Pisa, Via Cosimo Ridolfi, 10, I-56124 Pisa (PI), Italy

e-mail: m.sodini@ec.unipi.it

tel.: +390502216234; fax: +390502210603 
In addition, the idea that slight differences amongst producers' characteristics (e.g., heterogeneities in the cost function or different behaviours of players depending on the information set about the profit function) may cause major nonlinear local and global dynamic phenomena (such as lack of synchronisation and coexistence of attractors), has opened up the way for several interesting papers dealing with the behaviour of firms in a nonlinear oligopoly context (e.g., Tramontana et al. [26, 27]).

In this class of models, the cases of both linear demand (quadratic utility) and unit-elastic demand (Cobb-Douglas utility) are analysed with different objectives. The former assumption (linear demand) is illustrated by Kopel [18], Bischi et al. [6, 9] and Fanti et al. [16]. In particular, Kopel [18] shows that periodic and complex dynamics may take place in a model with cost functions that incorporate an inter-firm externality. Bischi et al. [9] concentrate on problems of synchronisation and intermittency that derive from a two-dimensional asymmetric map in a model with profit-maximising firms, while Fanti et al. [16] introduce managerial (relative profit) delegation and find that, despite the assumption of homogeneous players (symmetric map), a high degree of competition between managers may cause on-off intermittency, blow-out phenomena and multistability that are impossible under profit maximisation with homogeneous players. The latter assumption (unit-elastic demand) demand is illustrated by Puu [21, 23], Bischi et al. [6], Agliari et al. [2], Tramontana [25] and Gao et al. [17]. All these papers hypothesise unit-elastic demand on the part of consumers. In particular, Bischi et al. [6] show that symmetry-breaking bifurcations can occur only when some heterogeneities exist in the main parameters of the model, while Tramontana [25] explores a model characterised by one firm with complete knowledge of the profit function and myopic expectations about its rival's output decisions, and the other firm with no knowledge of the profit function that makes use of local estimation of its marginal profits to adjust production in the future period. The main finding of the paper is that the interior fixed point of a two-dimensional map may undergo either a flip bifurcation or Neimark-Sacker bifurcation.

The aim of the present paper is to characterise the local and global dynamic properties of a nonlinear Cournot duopoly with general isoelastic demand and price elasticity different from one (quasi-linear preferences) by assuming that firms use local estimates of marginal profits to adapt production decisions period by period. Unlike Bischi et al. [6], although players are homogeneous and thus the dynamics of the model is described by a two-dimensional symmetric map, we show that interesting global phenomena can be observed.

The rest of the paper is organised as follows. Section 2 describes the static model. Section 3 introduces a dynamic mechanism of production. Section 4 studies the properties of the basins of attraction. Section 5 introduces and describes critical curves. Section 6 studies local stability, global dynamic properties and synchronisation phenomena, and Section 7 concludes. The appendix provides microeconomic foundations for the general isoelastic demand function.

\section{The model}

We consider a Cournot duopoly with two types of agents: firms and consumers. A homogeneous good is produced and traded on a market with general isoelastic demand given by $p=Q^{\frac{-1}{\eta}}$ (which derives from quasi-linear preferences), ${ }^{1}$ where $p$ is the consumers' marginal willingness to pay for product $Q=q_{1}+q_{2}, q_{1} \geq 0$ and $q_{2} \geq 0$ are outputs produced by firms 1 and 2 , respectively, and $\eta>0$ is a parameter that captures the degree of demand

\footnotetext{
${ }^{1}$ See the appendix for microeconomic foundations of the general isoelastic demand function used in this paper.
} 
elasticity. The case $\eta=1$ (log-linear preferences) represents the unit-elastic demand model analysed, amongst others, by Bischi et al. [6], Puu [21] and Tramontana [25] in a Cournot duopoly under different hypotheses with regard to the information set of both players. In particular, Bischi et al. [6] assume that both players have limited information regarding profits (no knowledge of the market) and every firm behaves adaptively following a local estimate of their own profits (bounded rationality), Puu [21] assumes that that players have myopic expectations, both having complete knowledge of the profit function, while Tramontana [25] considers the heterogeneous case of myopic expectations and bounded rationality.

The production function of firm $i=\{1,2\}$ has constant marginal returns to labour, that is $q_{i}=L_{i}$, where $L_{i}$ represents the labour force employed by firm $i$ (Correa-López and Naylor [14]). Firm $i$ 's cost function is assumed to be $C_{i}\left(q_{i}\right)=c_{i} L_{i}=c_{i} q_{i}$, where $c_{1}$ and $c_{2}$, with $c_{1} \geq c_{2}>0$, represent the constant average and marginal costs of producing an additional unit of output for firm 1 and firm 2 , respectively.

Firm $i$ maximises profits $\left(\Pi_{i}\right)$ that are given by:

$$
\Pi_{i}=\left(p-c_{i}\right) q_{i}=\left(Q^{\frac{-1}{\eta}}-c_{i}\right) q_{i}=\left[\left(q_{i}+q_{-i}\right)^{\frac{-1}{\eta}}-c_{i}\right] q_{i} .
$$

We note that profit function (1) is concave in $q_{i}$ for every positive quantity chosen by firm $i$ 's rival. Profit maximisation by both firms yields:

$$
\begin{aligned}
& \frac{\partial \Pi_{1}}{\partial q_{1}}=0 \Leftrightarrow Q^{\frac{-1}{\eta}}-\frac{q_{1}}{\eta} Q^{\frac{-(1+\eta)}{\eta}}-c_{1}=0, \\
& \frac{\partial \Pi_{2}}{\partial q_{2}}=0 \Leftrightarrow Q^{\frac{-1}{\eta}}-\frac{q_{2}}{\eta} Q^{\frac{-(1+\eta)}{\eta}}-c_{2}=0,
\end{aligned}
$$

which implicitly define the reaction functions of firm 1 and firm 2 . The use of (2) and (3) allows us to obtain the unique Nash equilibrium of the game as follows:

$$
E^{*}=\left(q_{1}^{*}, q_{2}^{*}\right)=\left(Q^{*} \frac{c_{1}+\eta\left(c_{2}-c_{1}\right)}{c_{1}+c_{2}}, Q^{*} \frac{c_{2}+\eta\left(c_{1}-c_{2}\right)}{c_{1}+c_{2}}\right)
$$

where $Q^{*}:=\left(\frac{2 \eta-1}{\eta\left(c_{1}+c_{2}\right)}\right)^{\eta}$. The equilibrium values of price and profits are thus respectively given by:

$$
\begin{gathered}
p^{*}=\frac{\eta\left(c_{1}+c_{2}\right)}{2 \eta-1}, \\
\Pi_{1}^{*}=\frac{\left[\eta\left(c_{2}-c_{1}\right)+c_{1}\right]^{2}}{(2 \eta-1)\left(c_{1}+c_{2}\right)} Q^{*}, \quad \Pi_{2}^{*}=\frac{\left[\eta\left(c_{1}-c_{2}\right)+c_{2}\right]^{2}}{(2 \eta-1)\left(c_{1}+c_{2}\right)} Q^{*} .
\end{gathered}
$$

We note that if $c_{1}>c_{2}$ then $1 / 2<\eta<c_{1} /\left(c_{1}-c_{2}\right)$ should hold to guarantee that production, price and profits are positive.

If average (and marginal) costs are the same for both firms, that is $c_{1}=c_{2}=c$, then the Nash equilibrium of the game becomes the following:

$$
E^{*}=\left(q_{1}^{*}, q_{2}^{*}\right)=\left(\frac{Q^{*}}{2}, \frac{Q^{*}}{2}\right)
$$

where aggregate production now becomes $Q^{*}:=\left(\frac{2 \eta-1}{2 \eta c}\right)^{\eta}$. Then, the equilibrium values of 
price and profits boil down to: $p^{*}=\frac{2 \eta c}{2 \eta-1}$ and $\Pi_{1}^{*}=\Pi_{2}^{*}=\Pi^{*} \frac{c^{1-\eta}}{2(2 \eta-1)}\left(\frac{2 \eta-1}{2 \eta}\right)^{\eta}$. In this case, the condition $\eta>1 / 2$ is sufficient to guarantee that production, prices and profits are positive at the equilibrium. In addition, when $\eta=1$ (log-linear preferences) we find that $Q^{*}=1 /(2 c)$, $p^{*}=2 c$ and $\Pi^{*}=1 / 4$, i.e. profits are independent of average and marginal costs.

\section{Dynamics}

Time is discrete and indexed by $t=0,1,2, \ldots$. With regard to the information set of players, we assume that they have limited information (no knowledge of the market demand) as in Bischi et al. [6, 9] and Fanti et al. [16]. In order to overcome this informational lacuna, we assume that at any time $t$ player $i$ uses an adjustment mechanism based on local estimates of marginal profits at time $t\left(\partial \Pi_{i} / \partial q_{i}\right)$ to determine production at time $t+1$ (see Bischi and Naimzada [5] with regard to a model with discrete time, and Dixit [15] with regard to a model with continuous time):

$$
q_{i}^{\prime}=q_{i}+\alpha_{i} q_{i} \frac{\partial \Pi_{i}}{\partial q_{i}}
$$

where ' is the unit-time advancement operator, $\alpha_{i}>0$ is a coefficient that captures the speed of adjustment of firm $i$ 's quantity with respect to a marginal change in profits, and $\alpha_{i} q_{i}$ is the intensity of the reaction of player $i$. Therefore, firm $i$ increases or decreases production at time $t+1$ depending on whether $\partial \Pi_{i} / \partial q_{i}$ is positive or negative at time $t$. This type of adjustment mechanism implies that although players have incomplete information about demand and cost functions, they are assumed to be only able to get a local correct estimate of marginal profits in the current period.

By using (7), and taking into account the non-negativity of variables $q_{1}$ and $q_{2}$, the twodimensional dynamic system can be written as follows (Cánovas et al. [10]):

$$
T^{*}:\left\{\begin{array}{c}
q_{1}^{\prime}=\max \left\{0, q_{1}+\alpha_{1} q_{1}\left(Q^{\frac{-1}{\eta}}-\frac{q_{1}}{\eta} Q^{\frac{-(1+\eta)}{\eta}}-c_{1}\right)\right\} \\
q_{2}^{\prime}=\max \left\{0, q_{2}+\alpha_{2} q_{2}\left(Q^{\frac{-1}{\eta}}-\frac{q_{2}}{\eta} Q^{\frac{-(1+\eta)}{\eta}}-c_{2}\right)\right\}
\end{array},\right.
$$

However, in order to simplify the analysis in what follows we concentrate on the following map:

$$
T:\left\{\begin{array}{c}
q_{1}^{\prime}=q_{1}+\alpha_{1} q_{1}\left(Q^{\frac{-1}{\eta}}-\frac{q_{1}}{\eta} Q^{\frac{-(1+\eta)}{\eta}}-c_{1}\right) \\
q_{2}^{\prime}=q_{2}+\alpha_{2} q_{2}\left(Q^{\frac{-1}{\eta}}-\frac{q_{2}}{\eta} Q^{\frac{-(1+\eta)}{\eta}}-c_{2}\right)
\end{array},\right.
$$

with initial conditions that belong to $R_{+}^{2}=\left\{q_{1}, q_{2}: q_{1} \geq 0, q_{2} \geq 0\right\} \backslash\{0,0\}$. We remark that from a mathematical point of view map (9) is defined on set $D=\left\{q_{1}, q_{2}: q_{1}+q_{2}>0\right\}$. In addition, we define: 1) the set of interior feasible trajectories starting from initial conditions that belong to $\operatorname{int}\left(R_{+}^{2}\right)$ as $F=\left\{\left(q_{1}, q_{2}\right) \in \operatorname{int}\left(R_{+}^{2}\right): q_{1}^{\prime}>0, q_{2}^{\prime}>0, \forall n\right\}$, where $n$ is $n$th iterate of the map, and 2) the set of trajectories starting from initial conditions that belong to $R_{+}^{2}$ as $F^{*}=\left\{\left(q_{1}, q_{2}\right) \in R_{+}^{2}:\left(q_{1}^{\prime}, q_{2}^{\prime}\right) \in R_{+}^{2}, \forall n\right\}$. 
In what follows we analyse local and global dynamics in the symmetric case $c_{1}=c_{2}=c$ and $\alpha_{1}=\alpha_{2}=\alpha$ (homogeneous players). The following results hold.

Proposition 1. Let $\left(q_{1}, q_{2}\right) \in\left(D \backslash R_{+}^{2}\right)$. Then, $\left(q_{1}, q_{2}\right) \notin \operatorname{int}\left(R_{+}^{2}\right)$.

Proof. Let us consider the case $q_{1}<0$ and $q_{2}>0$. By contradiction, we assume $q_{1}^{\prime}>0$ and $q_{2}^{\prime}>0$. Then, from map (9) it follows that

$$
\left\{\begin{array}{l}
(1-\alpha c)+Q^{\frac{-1}{\eta}}<Q^{\frac{-1}{\eta}} \frac{q_{1}}{Q \eta} \\
(1-\alpha c)+Q^{\frac{-1}{\eta}}>Q^{\frac{-1}{\eta}} \frac{q_{2}}{Q \eta}
\end{array}\right.
$$

The second inequality in $(10)$ implies that $(1-\alpha c)+Q^{\frac{-1}{\eta}}>0$, but this is not consistent with the first inequality. The case $q_{1}>0$ and $q_{2}<0$ follows analogously. Q.E.D.

The previous result guarantees that trajectories starting from initial conditions on $R_{+}^{2}$ and converging to an attractor that lies on $\operatorname{int}\left(R_{+}^{2}\right)$ entirely lie on $\operatorname{int}\left(R_{+}^{2}\right)$. This means that it is not possible to have a trajectory that exits from $\operatorname{int}\left(R_{+}^{2}\right)$ and comes back on.

Proposition 2. If $\alpha c>1$ then $F$ is a bounded set. If $\alpha c<1$ and $\eta>1$ then infinity is not an attractor and all feasible trajectories are bounded. If $\alpha c<1$ and $1 / 2<\eta<1$ then infinity is not an attractor.

Proof. First of all, let us rewrite map (9) as follows

$$
T:\left\{\begin{array}{l}
q_{1}^{\prime}=q_{1}\left[(1-\alpha c)+Q^{\frac{-1}{\eta}}-Q^{\frac{-1}{\eta}} \frac{q_{1}}{Q \eta}\right] \\
q_{2}^{\prime}=q_{2}\left[(1-\alpha c)+Q^{\frac{-1}{\eta}}-Q^{\frac{-1}{\eta}} \frac{q_{2}}{Q \eta}\right]
\end{array},\right.
$$

If $\alpha c>1$, then $Q \rightarrow+\infty$ as $\left\|\left(q_{1}, q_{2}\right)\right\| \rightarrow+\infty$. By considering the sign of the three terms in brackets we obtain the existence of thresholds $\left(\hat{q}_{1}, \hat{q}_{2}\right)$ such that $q_{1}^{\prime}$ and $q_{2}^{\prime}$ are negative for $q_{1}>\hat{q}_{1}$ and $q_{2}>\hat{q}_{2}$.

If $\alpha c<1$ and $\eta>1$, the expression in brackets in both the first and second equations in (11) is positive. In fact, we note that by collecting $Q^{\frac{-1}{\eta}}$ in the second and third terms we obtain $Q^{\frac{-1}{\eta}}\left(1-\frac{q_{i}}{Q \eta}\right)>0$. In addition, such expression is less than one as $\left\|\left(q_{1}, q_{2}\right)\right\| \rightarrow+\infty$. This means that there exist thresholds $\left(\hat{\hat{q}}_{1}, \hat{\hat{q}}_{2}\right)$ sufficiently high such that $q_{1}^{\prime}<q_{1}$ and $q_{2}^{\prime}<q_{2}, \forall q_{1}>\hat{\hat{q}}_{1}$ and $\forall q_{2}>\hat{\hat{q}}_{2}$. At this point, it is sufficient to show that the expressions $q_{1}^{\prime}$ and $q_{2}^{\prime}$ take finite values in the compact set $\left[0, \hat{\hat{q}}_{1}\right] \times\left[0, \hat{\hat{q}}_{2}\right]$. We note that $q_{1}^{\prime}$ and $q_{2}^{\prime}$ are continuous functions and then they are bounded on the set of points $q_{1} \geq 0, q_{2}>0$ and $q_{1}>0, q_{2} \geq 0$, respectively. Then, we verify that they are bounded also as $\left(q_{1}, q_{2}\right) \rightarrow(0,0)$. Notice that $\forall\left(q_{1}, q_{2}\right) \in R_{+}^{2}$ : 


$$
\lim _{\left(q_{1}, q_{2}\right) \rightarrow(0,0)} q_{1}^{\prime}=\lim _{\left(q_{1}, q_{2}\right) \rightarrow(0,0)} q_{1}(1-\alpha c)+\lim _{\left(q_{1}, q_{2}\right) \rightarrow(0,0)} \frac{q_{1}}{Q^{\frac{-1}{\eta}}}\left(1-\frac{q_{1}}{Q \eta}\right)=0+0=0 .
$$

In fact, by recalling the non-negativity of $q_{1}$ and $q_{2}$ we get $\lim _{\left(q_{1}, q_{2}\right) \rightarrow(0,0)} \frac{q_{1}}{Q^{\frac{-1}{\eta}}}=0$ and $\left(1-\frac{q_{1}}{Q \eta}\right) \in[0,1]$

If $\alpha c<1$ and $1 / 2<\eta<1$, analogously with the previous case, the expression in brackets in both the first and second equations in (11) is less than one as $\left\|\left(q_{1}, q_{2}\right)\right\| \rightarrow+\infty$. This completes the proof. Q.E.D.

Remark 1. We note that in the case $\alpha c<1$ and $1 / 2<\eta<1$ we cannot exclude the existence of unbounded trajectories even if numerical simulations did not reveal this behaviour.

Remark 2. Assuming the same initial conditions, $q_{1}(0)$ and $q_{2}(0)$, trajectories that lie on the positive orthant of map $T$ are the same as those described by map $T^{*}$ for every $t$. In contrast, unfeasible trajectories of map $T$ coincide with trajectories that converge on the coordinate axes of map $T^{*}$ (Fanti et al. [16]).

Map $T$ defined in (9) has a unique interior fixed point with coordinates $E^{*}=\left(\frac{Q^{*}}{2}, \frac{Q^{*}}{2}\right)$ (which coincides with Nash equilibrium (4.2)), and three fixed points located on the invariant coordinate axes, that is: $O=(0,0), E_{1}=\left(\left(\frac{\eta-1}{c \eta}\right)^{\eta}, 0\right)$ and $E_{2}=\left(0,\left(\frac{\eta-1}{c \eta}\right)^{\eta}\right)$ when $\eta>1$. Fixed points $O, E_{1}$ and $E_{2}$ imply that at least one firm does not produce and exits the market.

Map $T$ is symmetric, i.e. it does not change if variables $q_{1}$ and $q_{2}$ are swapped, that is $T \circ S=S \circ T$, where $S:\left(q_{1}, q_{2}\right) \rightarrow\left(q_{2}, q_{1}\right)$. This implies that the diagonal $\Delta=\left\{\left(q_{1}, q_{2}\right): q_{1}=q_{2}\right\}$ is an invariant manifold, i.e. the dynamics lie on $\Delta$ for every $t$ by starting with $q_{1}(0)=q_{2}(0)$. In this case, the behaviour of the dynamic system is described by the restriction of map $T$ on $\Delta$, and synchronised trajectories (i.e., $q_{1}(t)=q_{2}(t)$ for every $t$ ) are governed by $T_{\Delta}: \Delta \rightarrow \Delta$, where

$$
T_{\Delta}: q^{\prime}=f(q):=q-\frac{1}{2} \alpha q\left(\frac{1-2 \eta}{(2 q)^{\frac{1}{\eta}} \eta}+2 c\right) .
$$

With regard to map $T_{\Delta}$, the following proposition can be established:

Proposition 3. (1) If $1 / 2<\eta<1$ and $\alpha<1 / c$ then the map is unimodal, convex and $\lim _{q \rightarrow 0^{+}} f(q)=+\infty, \lim _{q \rightarrow+\infty} f(q)=+\infty$.

(2) if $1 / 2<\eta<1$ and $\alpha>1 / c$ then the map is monotonically decreasing, convex and $\lim _{q \rightarrow 0^{+}} f(q)=+\infty, \lim _{q \rightarrow+\infty} f(q)=-\infty$. It implies that a threshold $\bar{q}=\frac{1}{2}\left(\frac{\alpha(2 \eta-1)}{2 \eta(\alpha c-1)}\right)^{\eta}>0$ exists such that $f(\bar{q})=0$.

(3) if $\eta>1$ and $\alpha<1 / c$ then the map is monotonically increasing, concave and 
$\lim _{q \rightarrow 0^{+}} f(q)=0, \lim _{q \rightarrow+\infty} f(q)=+\infty$.

(4) if $\eta>1$ and $\alpha>1 / c$ then the map is unimodal, concave and $\lim _{q \rightarrow 0^{+}} f(q)=0$, $\lim _{q \rightarrow+\infty} f(q)=-\infty$. It implies that a threshold $\bar{q}=\frac{1}{2}\left(\frac{\alpha(2 \eta-1)}{2 \eta(\alpha c-1)}\right)^{\eta}>0$ exists such that $f(\bar{q})=0$.

When the map is unimodal, we have a critical point

$$
q_{c r}=\frac{1}{2}\left(\frac{\alpha(2 \eta-1)(\eta-1)}{2 \eta^{2}(\alpha c-1)}\right)^{\eta}
$$

with a strictly positive image.

Proof. The proof is straightforward from the sign of both the first and second derivatives of (13) and the limits of $f$. Q.E.D.

Figure 1 exemplifies the results of Proposition 3 in a stylised way.
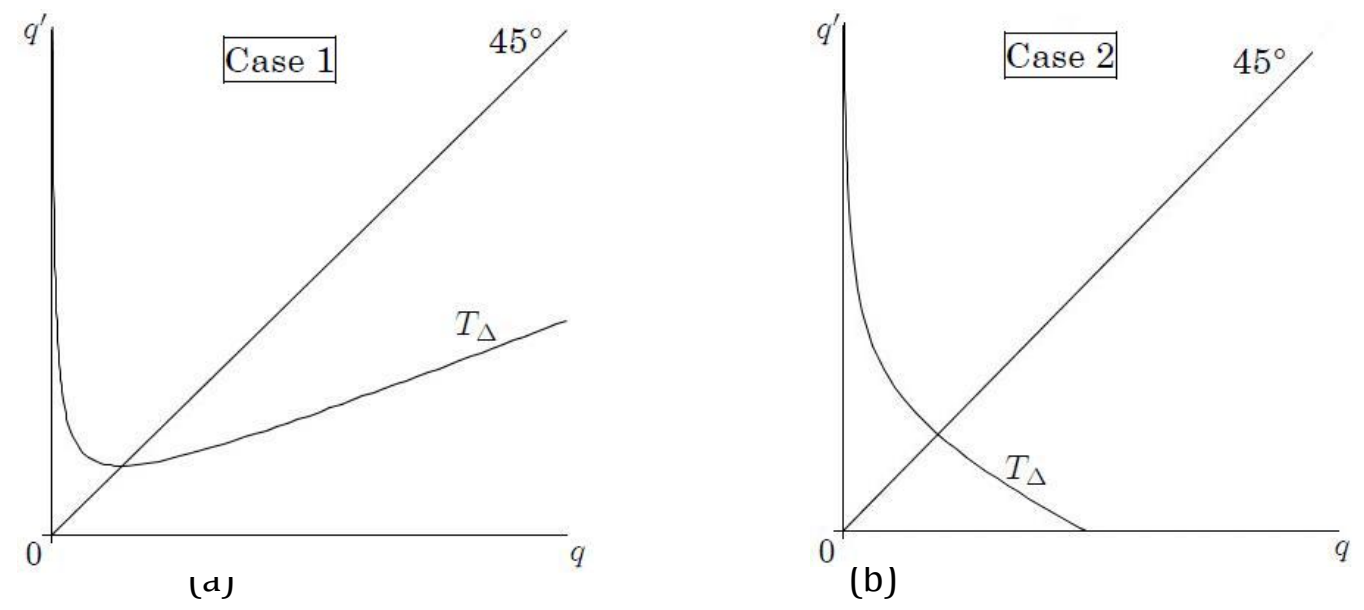

(b)
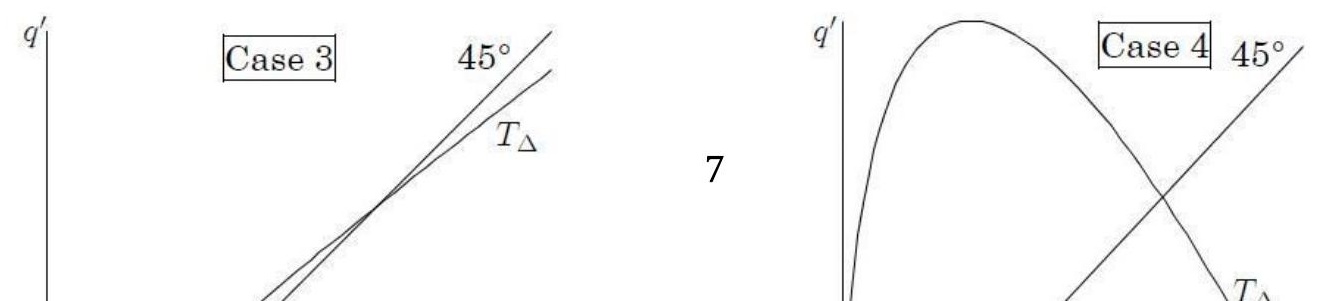
(c)

Figure 1. Different shapes of map $T_{\Delta}$ according to the classification of Proposition 3.

In order to study local stability of map $T_{\Delta}$ around $q^{*}=q_{1}^{*}=q_{2}^{*}$, it is sufficient to consider:

$$
f^{\prime}\left(q^{*}\right)=\frac{\eta-\alpha c}{\eta}
$$

From (15) it follows that the fixed point of $T_{\Delta}$ is locally stable for $\alpha<\frac{2 \eta}{c}$ and a flip bifurcation occurs at $\alpha=\alpha_{f l i p}:=\frac{2 \eta}{c}$.

Remark 3. With respect to the classification introduced in Proposition 3, we note that a flip bifurcation can occur if and only if $\alpha>1 / c$.

In the analysis that follows, we will concentrate on cases 3 and $4(\eta>1)$ because of the interesting dynamic events they can give rise to.

Assumption 1. $\eta>1$.

\section{Basins of attraction}

In this section we describe the properties of the basins of attraction of map (8). Given Remark 2 , we do this by analysing the properties of map (9). Following Bischi et al. [9] and Fanti et al. [16], it is possible to show that coordinate axes and their preimages of any rank form the boundary for initial conditions of any feasible trajectory. The dynamics of map (9) restricted to one of the axes is governed by the following one-dimensional map:

$$
q_{i}^{\prime}=s_{i}\left(q_{i}\right):=q_{i}+\alpha\left(q_{i}^{\frac{\eta-1}{\eta}} \frac{\eta-1}{\eta}-c q_{i}\right), \quad i=\{1,2\} .
$$

which has a positive fixed point $\widetilde{q}_{i}^{*}=\left(\frac{\eta-1}{\eta c}\right)^{\eta}$.

If $\alpha c<1$, then $s_{i}$ is an increasing, concave function and for any $q_{1}>0, q_{2}=0$ (resp. $q_{1}=0$, $q_{2}>0$ ) we have that $q_{1}^{\prime}>0, q_{2}^{\prime}=0$ (resp. $\left.q_{1}^{\prime}=0, q_{2}^{\prime}>0\right)$. 
If $\alpha c>1$, then $s_{i}$ is concave and unimodal. In this last case, let $\widetilde{q}_{i}^{\text {bound }}=\left(\frac{\alpha(\eta-1)}{\eta(\alpha c-1)}\right)^{\eta}$ and $\widetilde{q}_{i}^{c r}=\left(\left(\frac{1-\eta}{\eta}\right)^{2} \frac{\alpha}{\alpha c-1}\right)^{\eta}$ be the non-negative value of $q_{i}$ such that $q_{i}^{\prime}=0$ and the non-negative critical point of $s_{i}$, respectively. If $s_{i}\left(\widetilde{q}_{i}^{c r}\right)=\frac{(\eta-1)^{2 \eta-1}(\alpha c-1)^{1-\eta} \alpha^{\eta}}{\eta^{2 \eta}}<\widetilde{q}_{i}^{\text {bound }}$, i.e. if $\frac{(\eta-1)^{\eta-1}(\alpha c-1)}{\eta^{\eta}}>1$, then bounded trajectories along invariant axes are obtained provided that the initial value of the map lies on the segment line

$$
\omega_{i}=\left[O, O_{i}^{-1}\right],
$$

where $O_{i}^{-1}$ is the rank-1 preimage of the origin on the corresponding axis with a non-null coordinate equal to $\widetilde{q}_{i}^{\text {bound }}$. In contrast, unfeasible trajectories along the invariant axis are obtained by starting from an initial condition outside $\omega_{i}$. From these arguments and Proposition 1 it follows that $\omega_{i} \in F^{*}$.

In addition, the study of the Jacobian matrix restricted on $q_{i}$ axis (and evaluated at a generic point of it) reveals that the two eigenvalues associated with eigenvectors $(0,1)$ and $(1,0)$ are respectively the following: $\tilde{\lambda}_{1}=1+\frac{\alpha}{\eta}\left(q_{i}^{\frac{-1}{\eta}}\left(\frac{1}{\eta}-2+\eta\right)-c \eta\right)$ and $\tilde{\lambda}_{2}=1+\alpha\left(q_{i}^{\frac{-1}{\eta}}-c\right)$. The study of the former eigenvalue allows us to classify the dynamics along the axis. The study of the latter one allows us to show that trajectories starting close enough to the axes with positive initial conditions are repelled by the axes.

Proposition 4. If i) $\alpha c<1$ then any cycle on the positive semi axes is transversely repelling or ii) $\alpha c>1$ and $\frac{(\eta-1)^{\eta-1}(\alpha c-1)}{\eta^{\eta}}>1$ then any cycle on $\omega_{i}$ is transversely repelling.

Proof. Let us consider the properties along the axis $q_{1}\left(q_{2}=0\right)$. If $\alpha c<1$ then only a cycle of period one (fixed point) on the axis does exist and eigenvalue $\tilde{\lambda}_{2}$ evaluated on such a point is $\tilde{\lambda}_{2}^{*}=1+\frac{\alpha c}{\eta-1}>1$. Let us consider now the case $\alpha c>1$. In order to show the statement, we concentrate on the fact that eigenvalue $\tilde{\lambda}_{2}$ is decreasing with respect to $q_{i}$. We will show that $\tilde{\lambda}_{2}$ evaluated at the point $\left(\widetilde{q}_{1}^{*}, 0\right)$ is always greater than one. This implies that the eigenvalue associated with a cycle of any period will be greater than one. Let us consider, therefore, the eigenvalue $\tilde{\lambda}_{2}$ evaluated at the point $\left(\widetilde{q}_{1}^{\text {bound }}, 0\right)$, which is equal to $\frac{\alpha c-1}{\eta-1}$, and compare the inequalities $\frac{(\eta-1)^{\eta-1}(\alpha c-1)}{\eta^{\eta}}>1$ and $\frac{\alpha c-1}{\eta-1}>1$. Since the former inequality is more restrictive than the second one, the result follows. Q.E.D.

It thus follows that $\omega_{i}$ belongs to $\partial B(F)$ as well as to their preimages of any rank. Now, according to Bischi et al. [9], the next proposition holds. 
Proposition 5. Let $\alpha c>1$ and $\omega_{i}$ be the segment lines defined in (17). If $s_{i}\left(\widetilde{q}_{i}^{c r}\right)<\widetilde{q}_{i}^{\text {bound }}$. then,

$$
\partial B(F)=\left(\cup_{n=0}^{-\infty} T^{-n}\left(\omega_{1}\right)\right) \cup\left(\cup_{n=0}^{-\infty} T^{-n}\left(\omega_{2}\right)\right) .
$$

Proof. See Bischi et al. [9]. Q.E.D.

Proposition 5 may be specified by considering the theory of critical curves (Mira et al. [19]). Indeed, if $T^{-1}\left(\omega_{i}\right)$ belongs to regions without further preimages, then the feasible region is delimited by the segment lines $\omega_{i}$ and by their rank-1 preimages whose points can be obtained numerically (see Figure 4.b later in this paper).

For the subsequent analysis it will be useful to call attention to the theory of critical curves, which allows us to obtain the delimitation of a chaotic area when synchronization fails as well as study some global bifurcations.

\section{Critical curves}

It is important to note that map (9) is not an invertible endomorphism: for a given $\left(q_{1}^{\prime}, q_{2}^{\prime}\right)$ the rank-1 preimage may not exist or it may be multivalued. Given the assumption of isoelastic demand with price elasticity different from one, then in considering map (9) we have to solve a non-polynomial system to compute $\left(q_{1}, q_{2}\right)$ in terms of $\left(q_{1}^{\prime}, q_{2}^{\prime}\right)$. Nevertheless, through numerical computations and in accordance with models with unit-elastic demand or linear demand (e.g., Bischi et al. [6, 9]), the dynamic system (9) may have four, two or no real solutions (see Figure 2). Thus we can divide the plane into regions $Z_{0}, Z_{2}, Z_{4}$, according to the number of preimages (where the subscript in $Z$ indicates their number). A direct consequence of this fact is that, if we let $\left(q_{1}^{\prime}, q_{2}^{\prime}\right)$ vary in the plane $R^{2}$, the number of rank-1 preimages changes as the point $\left(q_{1}^{\prime}, q_{2}^{\prime}\right)$ crosses the boundary that separates these regions. Such boundaries are generally characterised by the existence of two coincident preimages. In this regard, following the notation used by Mira et al. [19], we introduce the definition of critical curves. The critical curve of rank-1, denoted by $L C$, is defined as the locus of points with two (or more) coincident rank-1 preimages located on a set called $L C_{-1}$. Since the map defined by (9) is continuously differentiable, $L C_{-1}$ belongs to the locus of points where the determinant of the Jacobian associated with map $T$ vanishes (i.e. the points where $T$ is not locally invertible), i.e.: $L C_{-1} \subseteq\left\{\left(q_{1}, q_{2}\right) \in R^{2}: \operatorname{Det}(J(T))=0\right\}$, and $L C$ is the rank-1 image of $L C_{-1}$ under $T$, i.e. $L C=T\left(L C_{-1}\right)$. We have omitted expressions of the Jacobian matrix and its determinant because they are cumbersome and do not define a classical known curve (as in Bischi and Lamantia [4]). However, by using the techniques introduced by Mira et al. [19] we can define through numerical calculations segments of critical curves of increasing rank to delimit trapping regions of the phase plane (see Mira et al. [19] for details and for applications on this topic).
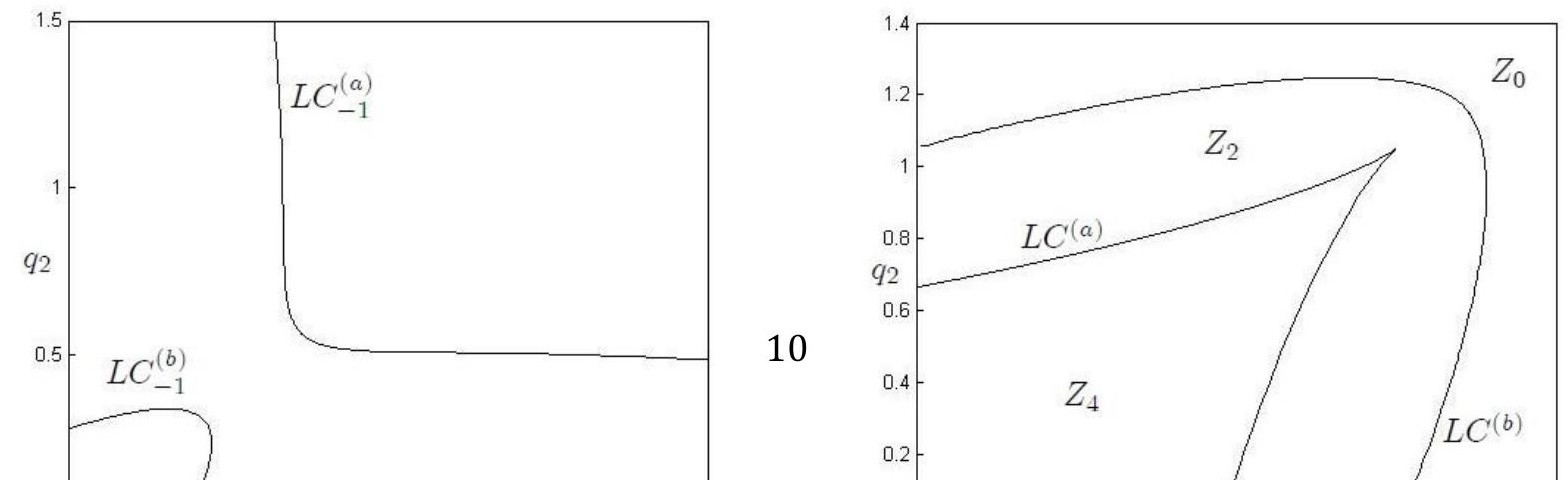
(a)

(b)

Figure 2. (a) Critical curves of rank- $0, L C_{-1}$, for map $T$. Parameter values: $\alpha=8, c=0.6$ and $\eta=2$. (b) Critical curves of rank-1, $L C=T\left(L C_{-1}\right)$, for the same parameter values as in panel (a). These curves separate the plane into regions $Z_{4}, Z_{2}$ and $Z_{0}$, whose points have a different number of preimages.

\section{Local analysis, global analysis and synchronisation}

The aim of this section is to study the dynamics of map (9). For doing this, we start with the local analysis around the diagonal. It is important to note that this topic is strictly related to synchronisation (intermittency). In this regard, we recall that dynamics are synchronised when there exists a $t^{*}$ such that $q_{1}(t)=q_{2}(t)$ for any $t>t^{*}$. It is therefore crucial now to study properties of the Jacobian matrix of map $T$ defined by (9).

By considering now the restriction of map $T$ on $\Delta$, we have the following Jacobian matrix:

$$
J(q, q)=\left(\begin{array}{cc}
l(q) & m(q) \\
m(q) & l(q)
\end{array}\right) .
$$

and the eigenvalues associated to a generic point on $\Delta$ :

$$
\lambda_{\|}=l(q)+m(q)=1-\alpha c+\frac{\alpha(\eta-1)}{\eta^{2}}\left(\eta-\frac{1}{2}\right)(2 q)^{\frac{-1}{\eta}},
$$

with eigenvector $(1,1)$ and

$$
\lambda_{\perp}=l(q)-m(q)=1-\alpha c+\frac{\alpha(\eta-1)}{\eta}(2 q)^{\frac{-1}{\eta}}
$$

with eigenvector $(1,-1)$.

The eigenvalue $\lambda_{\|}$is related to the invariant manifold $\Delta$ and coincides with the multiplier of the restriction of the map on $\Delta$. The eigenvector associated with the other eigenvalue is always orthogonal to $\Delta$ regardless of $q$.

Substituting the coordinates of $E^{*}$ we can characterise the local dynamics of the Nash equilibrium of the game by the following proposition.

Proposition 6. The fixed point $E^{*}$ is locally stable if and only if $\alpha<\frac{2 \eta}{c}$. For $\alpha=\frac{2 \eta}{c}, E^{*}$ undergoes a supercritical flip bifurcation. No other local bifurcation may arise around $E^{*}$.

Proof. From the symmetry of the map it follows that the eigenvalues are real (no Neimark Sacker bifurcation can occur). By substituting out the coordinates of $E^{*}$ in the generic expression of the eigenvalues of a point on $\Delta$ we have

$$
\lambda_{\|}\left(E^{*}\right)=1-\frac{\alpha c}{\eta}<\lambda_{\perp}\left(E^{*}\right)=1+\frac{\alpha c}{1-2 \eta}<1 .
$$

Hence the fixed point is stable if and only if $\lambda_{\|}\left(E^{*}\right)>-1 \Leftrightarrow \alpha<\frac{2 \eta}{c}$. Q.E.D. 
Remark 4. With regard to local stability properties around the interior fixed point, we note that $\eta$ plays a stabilising role. This means that an increase in $\eta$ (i.e., a reduction in the price elasticity of demand) favours, ceteris paribus, the local stability of $E^{*}$.

From an economic point of view, Remark 4 implies that the percentage variation in market demand that follows the percentage variation in price, is relatively low when $\eta$ is high (i.e., the market demand is sufficiently inelastic). This causes, ceteris paribus, 1) a relatively small change in marginal profits of every firm in the current period, and 2) a weak reaction of every firm in terms of quantity that will be produced in the future.

The analysis of Proposition 6 can be generalised when an attracting cycle for $T_{\Delta}$ on $\Delta$ exists. We now recall that for a $k$-cycle $\left\{\left(q_{1}, q_{1}\right), \ldots,\left(q_{m}, q_{m}\right)\right\}$ of $T$ embedded into the invariant line $\Delta$ and corresponding to the cycle $\left\{q_{1}, \ldots, q_{m}\right\}$ of $f$, the multipliers are given by

$$
\lambda_{\|}^{k}=\prod_{i=1}^{k}\left(l\left(q_{i}\right)+m\left(q_{i}\right)\right)
$$

with eigenvector $(1,1)$ and

$$
\lambda_{\perp}^{k}=\prod_{i=1}^{k}\left(l\left(q_{i}\right)-m\left(q_{i}\right)\right)
$$

with eigenvector $(1,-1)$.

The stability is ensured by the condition $\left|\lambda_{\|}^{k}\right|<1$. If we now consider a more complex situation, we recall that an attractor $A$ of $f$ is an asymptotically stable attractor of $T$ if and only if all trajectories belonging to $A$ are transversally attracting. In particular, if $A$ is a chaotic attractor a stability condition can be given in terms of the transverse Lyapunov exponent

$$
\Lambda_{\perp}=\lim _{n \rightarrow \infty} \sum_{i=1}^{n} \ln \left|\lambda_{\perp}(q(i))\right|,
$$

where $q(0) \in A$ and $q(i)$ is the trajectory generated by $f$.

The following classical definition of attractiveness can be established:

Definition 1. $A$ is an asymptotically stable attractor (or topological attractor) if it is Lyapunov stable, i.e. for every neighbourhood $U$ of $A$ there exists a neighbourhood $V$ of $A$ such that $T^{n}(V) \subset U$ for every $n \geq 0$ and the basin of attraction $B(A)$ contains a neighbourhood of $A$.

According to the initial conditions, it is possible to define a spectrum of Lyapunov exponents

$$
\Lambda_{\perp}^{\min }<\ldots<\Lambda_{\perp}^{\text {nat }}<\ldots \Lambda_{\perp}^{\max }
$$

where $\Lambda_{\perp}^{\text {nat }}$ is the Lyapunov exponent evaluated on a generic trajectory taken in the chaotic attractor. If a set is a Lyapunov attractor then $\Lambda_{\perp}^{\max }<0$. If $\Lambda_{\perp}^{\max }>0$ and $\Lambda_{\perp}^{\text {nat }}<0$, the set is no longer Lyapunov stable but it attracts a large set (the basin of attraction has positive Lebesgue measure) of points in the two-dimensional phase space. In this case, $A$ is a Milnor attractor. ${ }^{2}$ We recall here its formal definition.

\footnotetext{
${ }^{2}$ From a computational point of view it is difficult to prove that $A$ is a topological chaotic attractor, while it is easier to verify whether $A$ is a Milnor attractor (it is sufficient to show that a transversely repelling cycle exists with a negative natural Lyapunov exponent).
} 
Definition 2. A closed invariant set $A$ is said to be a weak attractor in Milnor sense if its stable set $B(A)$ has positive Lebesgue measure.

It is important to stress that a topological attractor is also a Milnor attractor, while the opposite is not true.

Finally If $\Lambda_{\perp}^{\text {nat }}>0$ then $A$ becomes a chaotic saddle and trajectories that start from initial conditions close to the diagonal can either be captured by an attractor that envelops the saddle or captured by other attractors.

In the next section we will see that in order to have highly nonlinear dynamics, we should have the coexistence of both an elastic demand (high values of $1 / \eta$ ) and a sufficiently high reactivity of each firm with respect to a marginal change in its profits $(\alpha)$.

\subsection{Numerical analysis}

The importance of the global analysis for economic models is recognised by the fact that restricting attention to the local behaviour of a map does not give information with regard to the structure of the basins of attraction and their qualitative changes when parameters vary. Since for economic models it is also important to understand the long-term behaviour of variables given initial conditions, a characterisation of the basins of attraction is indeed necessary if one wants to show phenomena that occur by starting from initial conditions far away from the fixed point or an attracting set.

We now turn to numerical analysis to explore the dynamic phenomena of a Cournot duopoly with isoelastic demand. In particular, in order to better characterise the possible local and global dynamic events of the model, we consider two different parameter sets with regard to both the average and marginal cost $(c)$ and price elasticity of demand $(\eta)$, and let the reactivity parameter $\alpha$ vary: 1) $c=0.6, \eta=2$ and 2) $c=0.5$ and $\eta=6$.

(1) We start numerical simulations by considering $c=0.6, \eta=2$ and $\alpha=8.15$. For these parameter values we observe that the dynamics are captured by a two-piece attractor on the diagonal (see Figure 3 panel a). Figure 3 panel b also shows that this is an attractor only in the Milnor sense. This means that several cycles embedded in the diagonal are transversely unstable and implies that trajectories approaching on the diagonal are burst away by transversely unstable cycles. Indeed, the convergence towards the unique chaotic (Milnor) attractor of the system embedded in the diagonal occurs only after a very long transient.
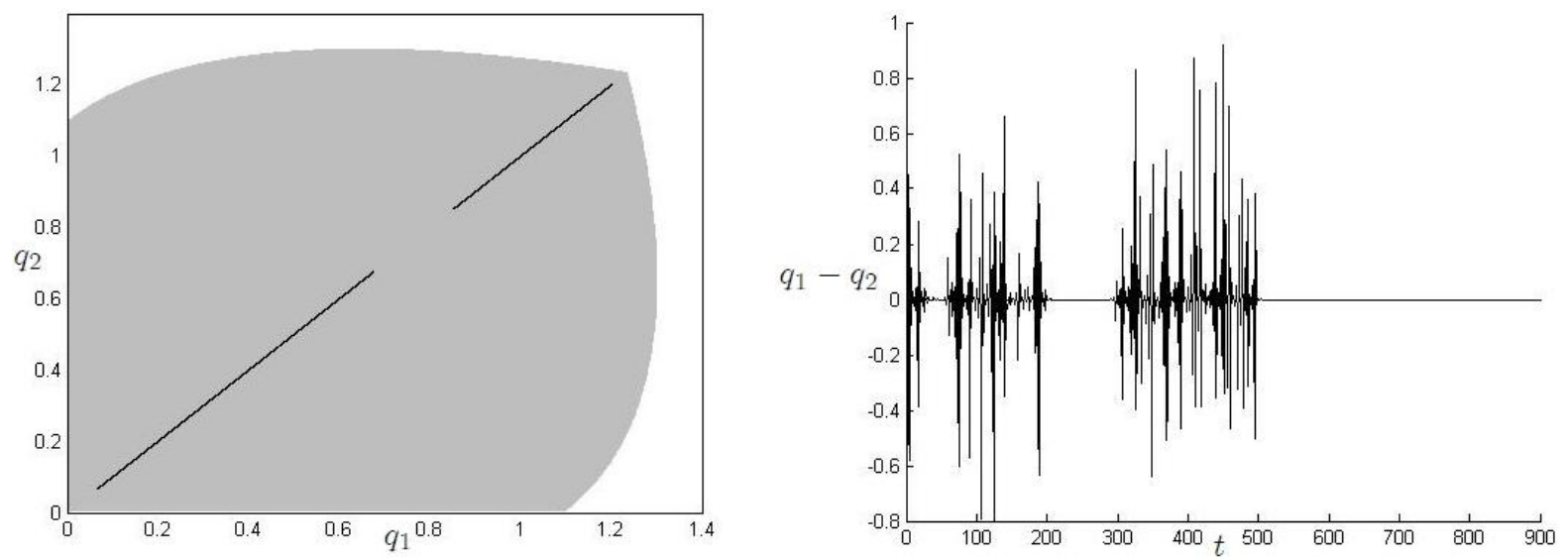
(a)

(b)

Figure 3. (a) A 2-piece Milnor attractor exists on the diagonal. (b) Synchronization occurs only after a long transient (on-off intermittency). Parameter set: $\alpha=8.15, c=0.6$ and $\eta=2$.

When $\alpha$ is reduced to 8 , an important change in long-term dynamics is observed. This is because synchronisation fails to occur and the dynamics of the model are no longer confined on the diagonal. Indeed, a two-dimensional chaotic attractor that governs long-term dynamics now exists (see Figure 4 panel a). In addition, Figure 4 panel b shows the boundary of the chaotic attractor by the use of the critical line technique, while the preimages of the segments $\omega_{i}$ show the borders of the basin of attraction of the interior fixed point. The fate of trajectories that start out from initial conditions that are positioned outside that region is to make at least one of the two firms exit from the market.

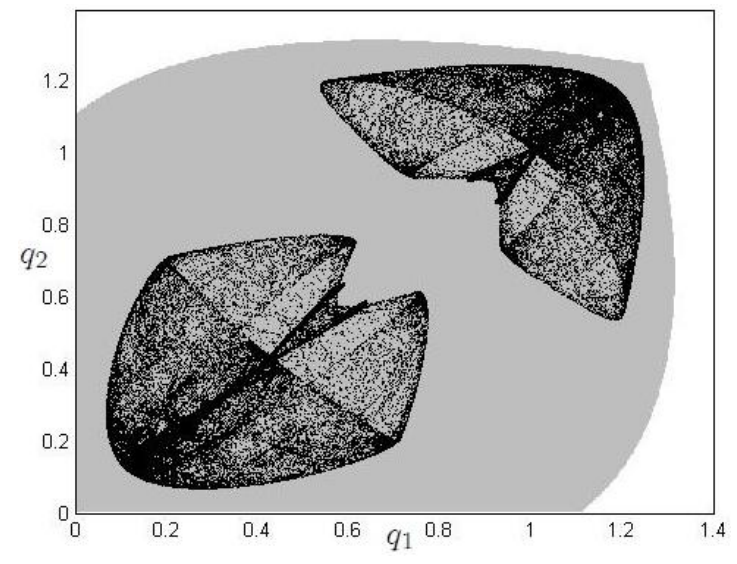

(a)

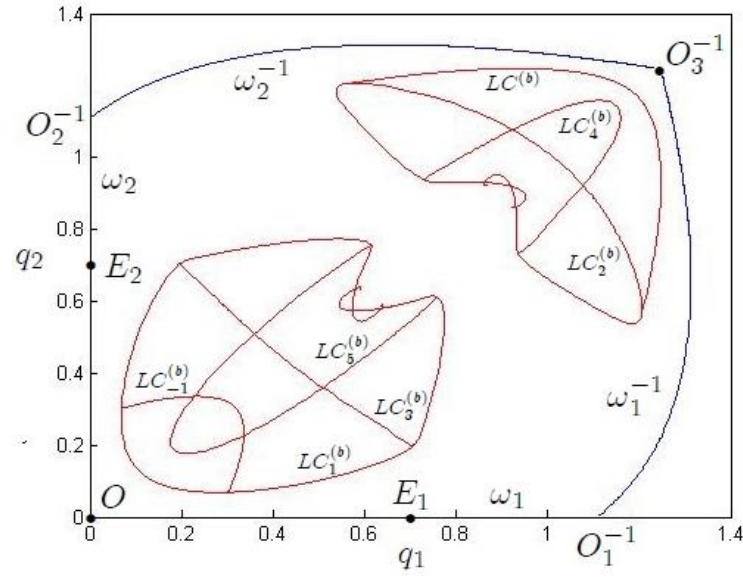

(b)

Figure 4. (a) Existence of a chaotic attractor for $\alpha=8, c=0.6$ and $\eta=2$. (b) Boundary of the attractor of $T$ for the same parameter values is represented by iterating six times a segment of curve $L C_{-1}$. The basin of attraction is bounded by the segments $\omega_{i}=\left[O, O_{i}^{-1}\right]$ and their rank-1 preimages (in this case $T^{-1}\left(\omega_{i}\right)$ belong to $\left.Z_{0}\right)$.

This change in long-term dynamics can be better understood through the study of the transverse Lyapunov exponent. The bifurcation diagrams plotted in Figure 5 shows the existence of a chaotic attractor on the diagonal for $\alpha \in[8,8.3]$ except for some windows of low-period cycles (corresponding to which the Lyapunov exponent plotted in the figure can be interpreted neither as the natural one nor a good approximation of it). We note however that in the range of the parameter $\alpha$ that we have considered, the Lyapunov exponent changes its sign several times (it eventually becomes positive for larger values of $\alpha$ not reported in the figure). This implies that small changes in the parameter can produce sharp changes in long-term dynamics. In other words, economies that start with similar initial conditions may end up looking very different.

In addition, Figure 5 also shows that the natural Lyapunov exponent at $\alpha=8$ is clearly positive such that synchronisation fails to occur. 

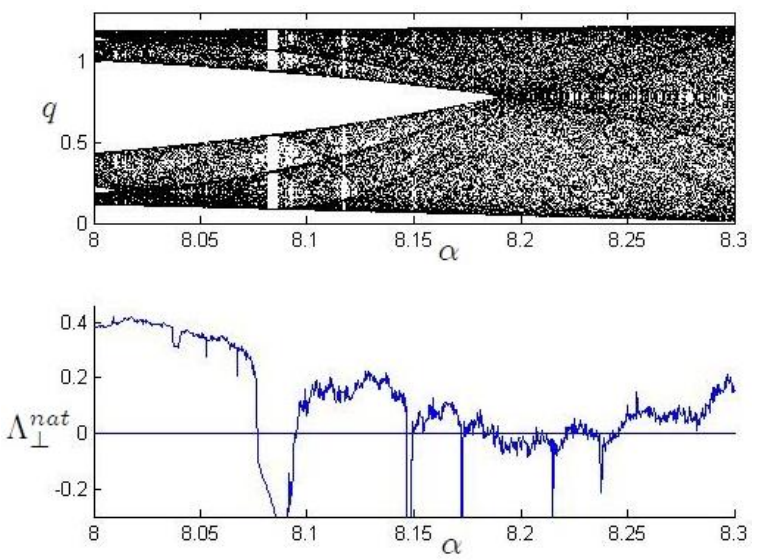

Figure 5. Bifurcation diagram of $T_{\Delta}$ and the corresponding transverse Lyapunov exponent for $\alpha \in[8,8.3]$ given $c=0.6$ and $\eta=2$.

(2) We now turn to the parameter set $c=0.5, \eta=6$ and let alpha vary from $\alpha=30.6$ and $\alpha=30.9$. When $\alpha=30.6$ we observe the coexistence of two interior chaotic attractors (Figure 6). The former attractor (à la Milnor) is the red one (the diagonal). The latter is a four-piece symmetric (with respect to the diagonal) chaotic attractor displaying an evolution similar to that described by Fanti et al. [16].

If we let $\alpha$ increase to 30.72 , we note that (i) only the attractors on the diagonal continue to exist (see Figure 7, panels a and b) and (ii) a basin bifurcation is close to occurring, as revealed by the position of curve $L C^{(b)}$ which is close to contact with $\partial B(F)$.

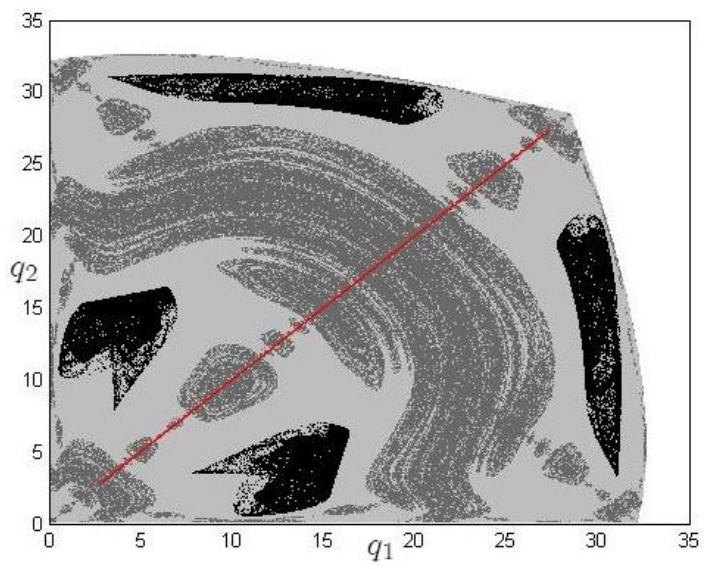

Figure 6. Coexistence of a chaotic four-piece attractor and of a Milnor attractor. Parameter set: $\alpha=30.6$, $c=0.5$ and $\eta=6$. 


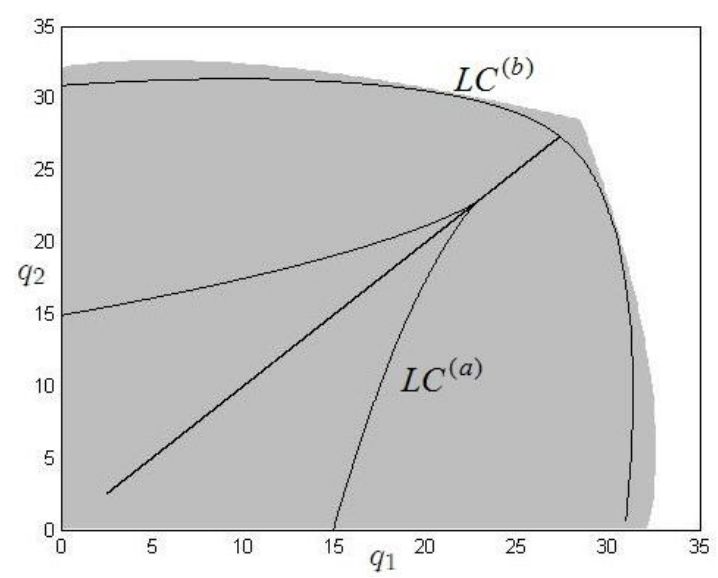

(a)

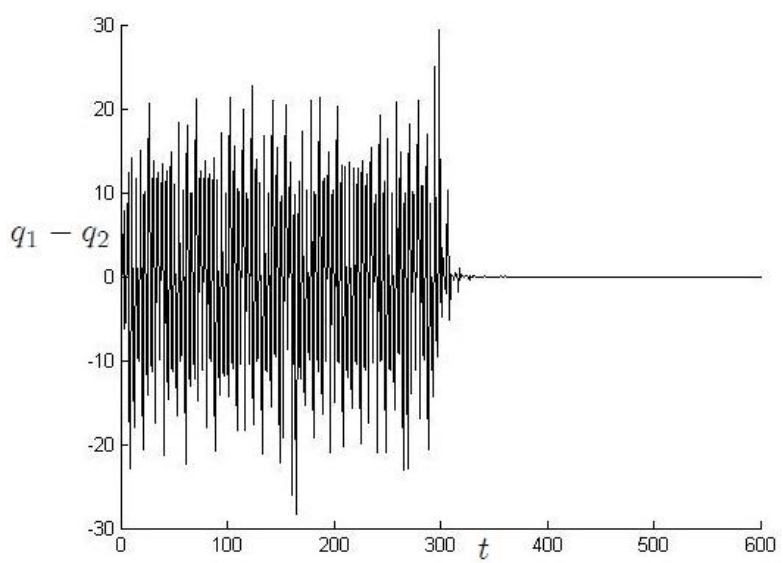

(b)

Figure 7. (a) A Milnor attractor exists on the diagonal. Critical curves show that a global bifurcation is close to occur. (b) Synchronization occurs only after a long transient. Parameter set: $\alpha=30.72, c=0.5$ and $\eta=6$.

When $\alpha=30.9$ global bifurcation has already occurred (see Figure 8 panel a) and infinitely many holes appear (see Figure 8 panel b). In this scenario two closely initial conditions can generate dramatically different trajectories from both a dynamic point of view in the long term and economic point of view (see Figure 9). Indeed, by starting for instance from initial conditions $q_{1}(0)=6, q_{2}(0)=10$ the two firms will tend to coordinate (synchronise) themselves to strictly positive values of production in the long term. In contrast with this, by slightly changing $q_{2}(0)$ from 10 to 10.1 , we observe that one of the two firms does not produce in the long term.

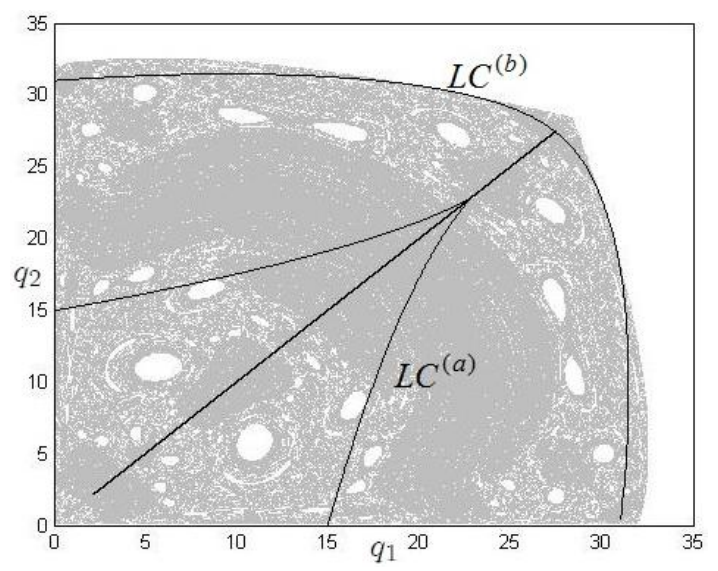

(a)

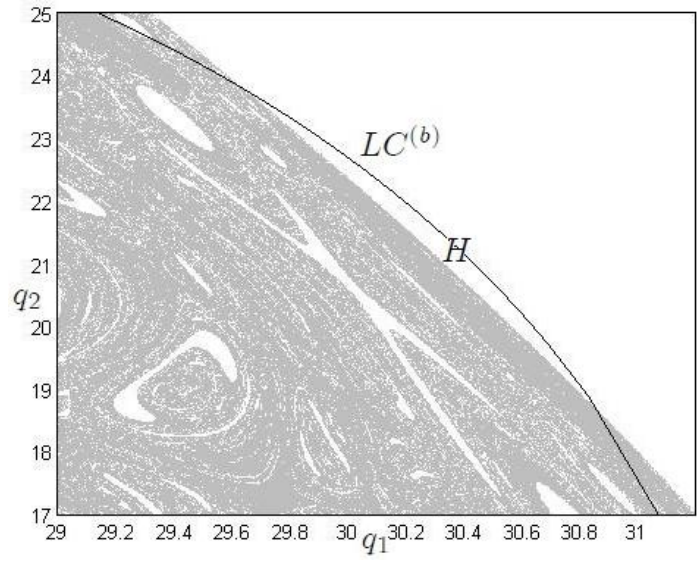

(b)

Figure 8. (a) Portions of the basin of attraction of trajectories that converge to invariant axes for map (8) enter $Z_{2}$ region. This causes the appearance of infinitely many holes in the basin of attraction of the Milnor attractor.

(b) Enlargement view of the birth of one of the principal holes $(H)$ in the basin of attraction of $A$. Parameter set: $\alpha=30.9, c=0.5$ and $\eta=6$. 


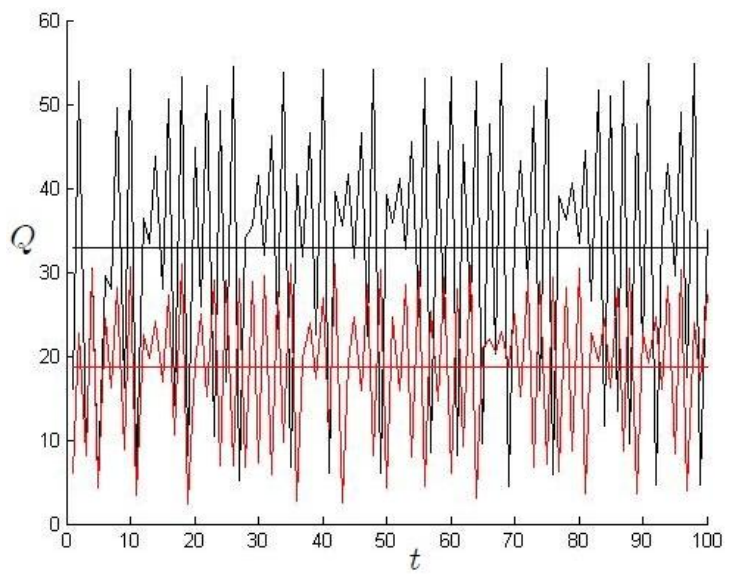

Figure 9. The figure shows how the appearance of holes in the basin of attraction of the attractor on the diagonal may cause difficulties in predicting feasible long-term trajectories of the model. In particular, two different trajectories generated by very closely initial conditions are depicted: one trajectory is associated with the coexistence of both firms in the market (black line); the other one is associated with the existence of only one firm in the market (red line), i.e. one of the two firm does not produce in the long-term. Parameter set: $\alpha=30.9, c=0.5$ and $\eta=6$. Initial conditions: $q_{1}(0)=6, q_{2}(0)=10$ (black line), $q_{1}(0)=6, q_{2}(0)=10.1$ (red line).

\section{Conclusions}

This paper analysed nonlinear dynamic phenomena in a Cournot duopoly with isoelastic demand and price elasticity different from one. The model is characterised by the hypothesis of homogeneous players: firms have the same cost function and production decisions in the future by both firms are taken by considering the steepest (local) slope of the profit function at the current state of production as in Bischi et al. [6,9].

The two-dimensional system describing the dynamics of the economy is a symmetric map. The existence of a price elasticity of demand different from one makes it possible to observe phenomena such as coexistence of attractors, coordination failures and complex structures of the basins of attraction, that are impossible in a model with homogeneous players and a unitelastic demand.

The papers most closely related to ours (with regard to the assumption of isoelastic demand) are Bischi et al. [6] and Bischi et al. [8]. The former considers the case of unit-elastic demand and players with incomplete information (and assumes local estimates of profit in the current period by firms), and show that to observe complex dynamic events it is essential to introduce some forms of heterogeneity in the main parameters of the model (e.g., different marginal costs). The latter paper explores the mechanism of adjustment of quantities over time, different from those based on local estimates of profits, called local monopolistic approximation. Unlike their works, we showed the importance of having a price elasticity of demand different from one in determining local and global phenomena in a nonlinear duopoly with quantity competition when players are homogeneous.

In addition, from an economic point of view, relaxing the assumption of unit-price elasticity of demand makes it possible to let equilibrium profits depend on average and marginal costs, while also introducing a major role or the elasticity parameter in determining the stability of the interior fixed point: ceteris paribus, policies aiming at reducing the price elasticity of demand may favour the stability of the equilibrium.

Acknowledgements performed within the
The authors gratefully acknowledge that this work has been activity of the PRIN-2009 project "Local interactions and global 
dynamics in economics and finance: models and tools", MIUR (Ministry of Education), Italy, and PRIN-2009 project "Structural change and growth", MIUR, Italy. Numerical simulations have benefited from algorithms that can be found in http://dysess.wikispaces.com/. We are indebted to Riccardo Cambini and two anonymous reviewers for insightful comments on an earlier draft. The usual disclaimer applies.

\section{Appendix. Microeconomic foundations of the general isoelastic demand function}

This appendix provides microeconomic foundations of the general isoelastic (inverse) demand function $p=Q^{\frac{-1}{\eta}}$, where $\eta>0$.

The economy is comprised of two types of agents: firms and consumers, and it is bisectorial, i.e. there exist a competitive sector that produces the numeraire good $y$ (whose price is normalised to one without loss of generality) and an oligopolistic sector with two firms (firm 1 and firm 2), each of which produces a homogeneous good $q_{i}, i=\{1,2\}$.

There exists a continuum of identical consumers whose preferences towards $q$ and $y$ are represented by the following quasi-linear utility function:

$$
V(Q, y)=U(Q)+y,
$$

where $Q=q_{1}+q_{2}$ and

$$
U(Q)=\left\{\begin{array}{ll}
\frac{\eta}{\eta-1} Q^{\frac{\eta-1}{\eta}} & \text { if } \quad \eta>0 . \\
\ln (Q) \quad \text { if } & \eta=1
\end{array} .\right.
$$

The representative consumer maximises utility function (27) subject to the budget constraint $p Q+y=M$, where $M>0$ is the exogenous nominal income of the consumer (assumed to be high enough to avoid the existence of corner solutions). Since $V(Q, y)$ is a separable function in variables $Q$ and $y$, and it is linear in $y$, there are no income effects on the duopolistic sector (see Singh and Vives [24] for a similar exercise with quadratic preferences and differentiated products).

Solving this problem gives the isoelastic inverse demand function:

$$
p=\left\{\begin{array}{lll}
Q^{\frac{-1}{\eta}} & \text { if } & \eta>0 . \\
Q^{-1} & \text { if } & \eta=1
\end{array} .\right.
$$

The literature on nonlinear oligopolies has generally used linear market demand functions (which derive from quadratic preferences) or unit-elastic demand functions, while leaving the study of nonlinear dynamic phenomena with general isoelastic demand essentially untreated (unlike the exception of Bischi et al. [8]). However, the static oligopoly literature has sometimes analysed models with isoelastic demands and price elasticity different from one with several purposes. Examples can be found in Chirco et al. [11], Collie [12], Colombo et al. [13], Neary [20] and more recently Beard [3]. In particular, Chirco et al. [11] develop a Cournot model with managerial incentive contracts (relative profits delegation) to study how the optimal delegation scheme is affected by the elasticity of market demand, while Beard [3] studies a Cournot oligopoly with $n$ profit-maximising firms and shows that the Nash equilibrium depends on both the elasticity of demand and share of industry costs.

\section{References}


[1] A. Agliari, C. Chiarella, L. Gardini, A re-evaluation of adaptive expectations in light of global nonlinear dynamic analysis, Journal of Economic Behavior \& Organization 60 (2006) 526552.

[2] A. Agliari, L. Gardini, T. Puu, The dynamics of a triopoly Cournot game, Chaos, Solitons and Fractals 11 (2000) 2531-2560.

[3] R. Beard, N-firm oligopoly with general iso-elastic demand, Bulletin of Economic Research (2013) forthcoming.

[4] G.I. Bischi, F. Lamantia, Chaos synchronization and intermittency in a duopoly game with spillover effects, in: T. Puu, I. Sushko, Eds., Oligopoly Dynamics: Models and Tools, Berlin: Springer, Chapter 8 (2002) 195-217.

[5] G.I. Bischi, A. Naimzada, Global analysis of a dynamic duopoly game with bounded rationality, in: J.A. Filar, V. Gaitsgory, K. Mizukami, Eds., Advances in Dynamics Games and Application, Boston: Birkhäuser Vol. 5 (2000) 361-385.

[6] G.I. Bischi, M. Gallegati, A. Naimzada, Symmetry-breaking bifurcations and representative firm in dynamic duopoly games, Annals of Operations Research 89 (1999) 253-272.

[7] G.I. Bischi, C. Mammana, L. Gardini, Multistability and cyclic attractors in duopoly games, Chaos, Solitons \& Fractals 11 (2000) 543-564.

[8] G.I. Bischi, A. Naimzada, L. Sbragia, Oligopoly games with Local Monopolistic Approximation, Journal of Economic Behavior \& Organization 62 (2007) 371-388.

[9] G.I. Bischi, L. Stefanini, L. Gardini, Synchronization, intermittency and critical curves in duopoly games, Mathematics and Computers in Simulation 44 (1998) 559-585.

[10] J.S. Cánovas, T. Puu, M. Ruíz, The Cournot-Theocharis problem reconsidered, Chaos, Solitons \& Fractals 37 (2008) 1025-1039.

[11] A. Chirco, M. Scrimitore, C. Colombo, Competition and the strategic choice of managerial incentives: the relative performance case, Metroeconomica 62 (2011) 533-547.

[12] D. Collie, Collusion and the elasticity of demand, Economics Bulletin 12 (2004) 1-6.

[13] C. Colombo, A. Chirco, M. Scrimitore, Strategic delegation and market competitiveness, Economics Bulletin 29 (2009) 1708-1716.

[14] M. Correa-López, R.A. Naylor, The Cournot-Bertrand profit differential: a reversal result in a differentiated duopoly with wage bargaining, European Economic Review 48 (2004) 681696.

[15] A.K. Dixit, Comparative statics for oligopoly, International Economic Review 27 (1986) 107-122.

[16] L. Fanti, L. Gori, M. Sodini, Nonlinear dynamics in a Cournot duopoly with relative profit delegation, Chaos, Solitons \& Fractals 45 (2012) 1469-1478.

[17] X. Gao, W. Zhong, Z. Mei, Nonlinear Cournot oligopoly games with isoelastic demand function: the effects of different behavior rules, Communications in Nonlinear Science and Numerical Simulation 17 (2012) 5249-5255.

[18] M. Kopel, Simple and complex adjustment dynamics in Cournot duopoly models, Chaos, Solitons \& Fractals 12 (1996) 2031-2048.

[19] C. Mira, L. Gardini, A. Barugola, J.C. Cathala, Chaotic Dynamics in Two-Dimensional Noninvertible Maps, Singapore: World Scientific Publishing (1996).

[20] J.P. Neary, International trade in general oligopolistic equilibrium, CESifo Area conference on global economy (2009).

[21] T. Puu, Chaos in duopoly pricing, Chaos, Solitons \& Fractals 1 (1991) 573-581.

[22] T. Puu, Complex dynamics with three oligopolists, Chaos, Solitons \& Fractals 7 (1996) 2075-2081.

[23] T. Puu, The chaotic duopolists revisited, Journal of Economic Behavior \& Organization 33 (1998) 385-394. 
[24] N. Singh, X. Vives, Price and quantity competition in a differentiated duopoly, RAND Journal of Economics 15 (1984) 546-554.

[25] F. Tramontana, Heterogeneous duopoly with isoelastic demand function, Economic Modelling 27 (2010) 350-357.

[26] F. Tramontana, L. Gardini, T. Puu, Cournot duopoly when the competitors operate multiple production plants, Journal of Economic Dynamics and Control 33 (2009) 250- 265.

[27] F. Tramontana, L. Gardini, T. Puu, Global bifurcations in a piecewise-smooth Cournot duopoly game, Chaos, Solitons \& Fractals 43 (2010) 15-24. 